\title{
SARS-CoV-2 transmission by aerosols: an underestimated question?
}

\author{
Luciene Muniz Braga* (1), Oswaldo Jesus Rodrigues da Motta ${ }^{1,2}$ (D), Andréia Patrícia Gomes (1),

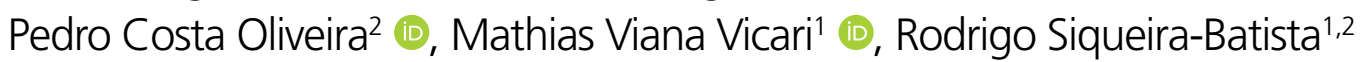

KEYWORDS: Coronavirus infections. Betacoronavirus. Aerosols.

Dear editor,

The actual pandemic caused by SARS-CoV-2 (Severe Acute Respiratory Syndrome), an etiological agent of COVID-19 (Coronavirus Disease 2019), has already infected more than 16 million people in the world, causing about 900,000 deaths in September 2020. The pathogen belongs to a group of microorganisms that infect the lower and upper airways and cause respiratory diseases, from milder forms resembling a common cold, to classic cases of influenza syndrome - with the symptoms of cough, fever, and coryza - to more serious situations, with pneumonia and severe acute respiratory syndrome (SARS) ${ }^{1}$.

Initially, it was believed that the transmission of the virus occurred mainly through droplets and contact, considering the transmission by aerosols restricted to specific situations of medical practice such as nebulization, orotracheal intubation, cardiorespiratory resuscitation, and bronchoscopy, among others. More recent studies, however, have shown the importance of aerosol transmission outside the health environment, including reports of virus spread in concerts, elevators, and shopping centers ${ }^{2}$.

To understand the dynamics of transmission of SARS-CoV-2 it is necessary to differentiate between droplets and aerosols, two vehicles for the respiratory dissemination of microorganisms that are produced especially by speaking, coughing, or sneezing. The droplets are larger than 5 micrometers. Infection may occur when droplets reach the oral, nasal, and/or ocular mucosa of the susceptible. It is also important to comment that when these droplets are deposited on surfaces, they make transmission by contact possible, from fomites or the surface of a body contaminated by the viral agent, taken later to the mucous membranes $^{1,3}$. In objects made of steel and plastic, the virus has been stable for up to 72 hours, with high positivity for SARS-CoV-2 in devices such as garbage cans and bed grids ${ }^{4}$. Considering the size of the droplets and their range, distancing of more than 1.5 meters between people, and frequent sanitization of the hands (minimizing the propagation by contact), are important guidelines in terms of biosafety ${ }^{1,4}$.

Aerosols, with a diameter of less than 5 micrometers, are transmitted by air. According to the physical-mathematical foundations of fluid dynamics, such particles are carried inside a cloud of turbulent gas and may remain suspended in the air for long periods. In this case, the description of the phenomenon is based on Brownian Motion behavior, which is explained by the collisions of the particles with the suspended molecules and fluid viscosity. Thus, a chaotic and random movement occurs on the aerosols, which are carried by airflow, distributing themselves in different areas of the environment ${ }^{5,6,7}$. Based on what had been described in the epidemics triggered by other coronaviruses, such as SARS-CoV and MERS-CoV, there already existed suspicion that the spread of SARS-CoV-2 was occurring using aerosols $\mathrm{s}^{5,7}$.

Given the high transmissibility of SARS-CoV-2 - especially due to the possible underestimation of virus dispersion by aerosols $\mathrm{s}^{4-8}-$ it is essential to review individual protection measures, in terms of the recommendation of universal use of the N95 respirator or equivalent (PFF2), especially in situations of health care units treating patients with respiratory symptoms. Furthermore, in the current context of the COVID-19 pandemic, and this is the proposal of the present manuscript, the universal use of the N95 or PFF2 respirator should be considered for all healthcare professionals who provide direct care to patients in closed environments - a characteristic of most healthcare

'Universidade Federal de Viçosa - Viçosa (MG), Brazil.

2Faculdade Dinâmica do Vale do Piranga - Ponte Nova (MG), Brazil.

*Corresponding author: luciene.muniz@ufv.br

Conflicts of interest: the authors declare there are no conflicts of interest. Funding: none. Received on September 25, 2020. Accepted on September 30, 2020. 
institutions - due to the possibility of aerosol formation and the low possibility of air exchange.

In addition, a cost-benefit analysis of the medical mask (which should be replaced every three hours at the most, or sooner if it is wet) compared to the N95 respirator is pertinent for health care professionals, considering the time of use of this equipment and the related costs. In fact, the availability of the latter (N95 respirator) may even be less costly - from a purely financial point of view - than the offer of medical masks.

It should also be considered that the number of asymptomatic and pre-symptomatic patients and health professionals, who present SARS-CoV-2 detectable in the secretions of the oropharynx and/or nasopharynx, allows the transmission to those who are in the same environments, both workers and patients. In this situation, it is imprudent to act in a risk establishment logic, limiting the use of N95 respirators or equivalent only when it is believed that there will be an aerosol-generating procedure in the classical ways. The risk of contagion, infection and illness of health professionals is aggravated by these findings and is associated with the time of exposure to the patient, the degree of agglomeration in poorly ventilated places and the quality of air in the environment ${ }^{1,5}$. In fact, the existence of (1) adequate air exchange, (2) filtration with HEPA (HighEfficiency Particulate Arrestance) filter type decontamination unit, (3) exhaust system and rooms with negative pressure and (4) use by the professional of the adequate protective equipment, which in this case, certainly should not be the medical mask but the N95 respirator or equivalent (PFF2) must be taken into consideration? ${ }^{7}$.

It is worth noting, in this context of increased risk of aerosol transmission, that investment in technologies such as ultraviolet radiation-based air disinfectants such as $U V$ germicidal upper-room has shown good results against SARS-CoV-2. For the general population, cloth masks are a viable option, if it is not possible to use medical procedure masks or respirators, and it is necessary to evaluate the type of cloth, the number of layers and the presence of a filter, the change time (every three hours or before in case of humidity), the fitting to the nasal and mento-mandibular anatomy and water resistance ${ }^{8-10}$. The use of a medical mask is currently recommended. To improve performance and to reduce SARS-CoV-2 transmission and exposure, the CDC suggests the combination of the cloth mask covering the medical procedure mask, double mask, or the use of respirators ${ }^{10}$.

To have good results in the control of SARS-CoV-2, (inter) national health policymakers and administrators of health institutions must ensure the necessary working conditions in primary, secondary, and tertiary health care - with (1) the application of protocols to verify the existence of symptoms, in the health unit workers, before the beginning of the working day and (2) the guarantee of personal protection equipment for the performance of care activities and high levels of vaccine for all. In addition, it is essential to allocate resources to inform the population about prevention measures, the effectiveness of the mask and the importance of wearing it - even in the absence of symptoms - and the correct way to sanitize hands, clothes, and other surfaces ${ }^{9}$. The duration of vaccine protection is not yet known and widespread vaccination to reach an appropriate level of population-level immunity (60-70\%) will take some time. Therefore, the use of masks will have to continue until the cessation of the pandemic. The victory over COVID-19 will depend on these efforts.

\section{AUTHORS" CONTRIBUTIONS}

LMB: Conceptualization, Supervision, Writing - Original Draft, Writing - Review \& Editing. OJRM: Formal Analysis, Writing - Original Draft, Writing - Review \& Editing. APG: Conceptualization, Supervision, Writing - Review \& Editing. PCO: Writing - Original Draft. MVV: Writing Original Draft, Formal Analysis. RSB: Conceptualization, Supervision, Writing - Review \& Editing.

\section{REFERENCES}

1. Centers for Disease Control and Prevention. Coronavirus (COVID19). Atlanta: Centers for Disease Control and Prevention; 2020. [cited on Aug. 08, 2020]. Available from: https://www. cdc.gov/coronavirus/2019-ncov/index.html

2. Cai J, Sun W, Huang J, Gamber M, Wu J, He G. Indirect virus transmission in cluster of COVID-19 cases, Wenzhou, China, 2020. Emerg Infect Dis. 2020;26(6):1343-5. https://doi. org/10.3201/eid2606.200412

3. Leung NHL, Chu DKW, Shiu EYC, Chan KH, McDevitt JJ, Hau BJP, et al. Respiratory virus shedding in exhaled breath and efficacy of face masks. Nat Med. 2020;26(5):676-80. https:// doi.org/10.1038/s41591-020-0843-2
4. Guo ZD, Wang ZY, Zhang SF, Li X, Li L, Li C, et al. Aerosol and surface distribution of severe acute respiratory syndrome coronavirus 2 in Hospital Wards, Wuhan, China, 2020. Emerg Infect Dis. 2020;26(7):1583-91. https://doi.org/10.3201/ eid2607.200885

5. Wilson NM, Norton A, Young FP, Collins DW. Airborne transmission of severe acute respiratory syndrome coronavirus-2 to healthcare workers: a narrative review. Anaesthesia. 2020;75(8):1086-95. https://doi.org/10.1111/anae.15093

6. Verma S, Dhanak M, Frankenfield J. Visualizing the effectiveness of face masks in obstructing respiratory jets. Phys Fluids (1994). 2020;32(6):061708. https://doi.org/10.1063/5.0016018 
7. Nardell EA, Nathavitharana RR. Airborne spread of SARS-COV-2 and a potential role for air disinfection. JAMA. 2020;324(2):141-2. https://doi.org/10.1001/ jama.2020.7603

8. Chughtai AA, Seale H, Macintyre CR. Effectiveness of cloth masks for protection against severe acute respiratory syndrome coronavirus 2. Emerg Infect Dis. 2020;26(10):e200948. https:// doi.org/10.3201/eid2610.200948
9. Klompas M, Morris CA, Sinclair J, Pearson M, Shenoy ES. Universal masking in hospitals in the COVID-19 era. N Engl J Med. 2020;382(21):e63. https://doi.org/ 10.1056/NEJMp2006372

10. Brooks JT, Beezhold DH, Noti JD, Coyle JP, Derk RC, Blachere FM, Lindsley WG. Maximizing Fit for Cloth and Medical Procedure Masks to Improve Performance and Reduce SARS-CoV-2 Transmission and Exposure, 2021. MMWR Morb Mortal Wkly Rep 2021; 70:254-257. http://dx.doi.org/10.15585/mmwr.mm7007e1 